\title{
Comentario
}

\section{5: EL AÑO INTERNACIONAL DE LOS SUELOS}

\author{
Floria Bertsch ${ }^{1 / *}$, Carlos Henríquez*
}

Palabras clave: Suelo; funciones ecosistémicas; año internacional.

Keywords: Soil; ecosystem functions; international year.

\section{RESUMEN}

Este comentario resulta pertinente por considerarse que la celebración del Año Internacional de los Suelos durante el 2015, con certeza, posicionará al recurso suelo en la dimensión que le corresponde dentro de la agricultura sostenible a nivel mundial. Asimismo, este acontecimiento coincide con la conmemoración del 60 Aniversario del Centro de Investigaciones Agronómicas de la Universidad de Costa Rica, en donde se ha realizado la mayor parte de la investigación edafológica del país. En el comentario se efectúa un análisis de las funciones ecosistémicas del suelo, las acciones mundiales que se están llevando a cabo para lograr ese posicionamiento y concretamente, las actividades locales que se realizaron durante este año en nuestro país, con el fin de lograr que esta visión integral del suelo sea la que conduzca a una convivencia más exitosa de sus funciones y así, a una verdadera conservación del recurso.

\section{ABSTRACT}

2015: International Year of Soils. Certainly, the celebration of the International Year of Soils during this 2015 will result in the soil resource positioning within sustainable agriculture worldwide. As this event coincided with the commemoration of the 60th Anniversary of the Centro de Investigaciones Agronómicas of Universidad de Costa Rica, where has done most of the soils research in the country, was considered right to do an analysis of the soil ecosystem functions, a review of global actions being undertaken to achieve this positioning. Also, are included the local activities carried out during this year in order to achieve this comprehensive view of soil. This approach will lead to a more successful coexistence of its functions and thus, a true soil conservation.
Autor para correspondencia. Correo electrónico: accs.cia@ucr.ac.cr
Centro de Investigaciones Agronómicas, Asociación Costarricense de la Ciencia del Suelo. Costa Rica. 
En este año 2015, nos llegó por fin el turno de valorar un recurso de suma importancia para el planeta: ¡el suelo!

La Asamblea General de las Naciones Unidas en su Sesión $\mathrm{N}^{\circ}$. 68, declaró al 2015 como el Año Internacional del Suelo. Esta acción simbólica de convocar al mundo entero a celebrar la importancia del suelo para la vida es, en términos prácticos, "ponerle la pata que le faltaba al banco" de la sostenibilidad. Ahora hablamos del suelo, el agua y el aire, como los 3 recursos imprescindibles para la vida en la Tierra.

$Y$ es que para nadie es un secreto que a pesar de su importancia para los servicios ecosistémicos y la vida del ser humano, el suelo ha sido, dentro de los recursos naturales, el menos considerado y valorado, el más olvidado. Desde que inició la corriente de pensamiento agroecológico y de Agricultura Sostenible comenzó a ser claro en la comunidad científica que los sistemas biológicos funcionan como unidades integrales, sea un bosque natural, una plantación de café, un sistema agrosilvopastoril, y que dentro de éstos, el suelo constituye un componente clave. No obstante, fuera del sector académico y profesional, la percepción del mismo como recurso vital y relevante para la vida del planeta ha sido verdaderamente limitada.

El suelo es un recurso finito y frágil, que se encuentra bajo una presión creciente y que se puede perder en forma permanente e inexorable con mucha facilidad si no se maneja apropiadamente. Su formación requiere cientos y hasta miles de años por lo que su pérdida y deterioro es algo que no podemos permitir.

Por ejemplo, se dice que para formar una capa de un centímetro de suelo se requieren entre 800 y 3000 años, y esa cantidad de suelo en condiciones desprotegidas, puede desaparecer en un solo aguacero.

FAO ha hecho cálculos que documentan que anualmente se pierden en el mundo 50.000 $\mathrm{km}^{2}$ de suelo, o sea una extensión similar a la de nuestro país.

Costa Rica tiene la particularidad de que a pesar de ser un país pequeño, comparativamente a otros del continente y del mundo, su diversidad en topografía, clima y materiales parentales, ha hecho que tenga igualmente una variabilidad de suelos muy grande.

Esto permite la existencia de gran biodiversidad que a su vez propicia una amplia gama de sistemas agropecuarios y por ende, una mayor vulnerabilidad a la degradación y pérdida de suelos.

Las pendientes pronunciadas, así como la alta pluviosidad, son sin duda 2 factores naturales que propician la erosión; estos factores aunados a descuidos en la construcción de caminos, manejo de suelos sin cobertura y técnicas de labranza inadecuadas, son una combinación desastrosa para el recurso suelo. Otros problemas como compactación por sobrepastoreo, diferentes tipos de contaminación y exceso de fertilización, se unen a la lista de causas que degradan y repercuten en la pérdida de calidad del recurso suelo.

De los 6 grandes sistemas que conforman nuestra Tierra, a saber, la atmósfera (la capa gaseosa que nos rodea), la hidrosfera (el agua), la criosfera (las áreas congeladas), la biosfera (todo el mundo vivo dentro del que nos incluimos), la litosfera (las rocas que dan forma al planeta), y la pedosfera (que incluye al suelo), es precisamente éste último, el menos reconocido como fundamental, tanto por el individuo común como por los órganos internacionales en quienes recaen las decisiones globales.

$\mathrm{Si}$ tratamos de analizar la causa de este fenómeno podemos enumerar algunas posibilidades.

En el diario vivir, tomamos agua pero no comemos suelo directamente; recibimos los rayos del sol y sentimos si el calor es más o menos intenso; recordamos si hemos usado la sombrilla o no, o sea estamos conscientes del clima. Las funciones del suelo, por el contrario, aunque vitales no están presentes en nuestra cotidianeidad, más bien, lo que hacemos sobre el suelo diariamente, sin darnos cuenta, es "pisotearlo" cada vez que caminamos. Si ponemos más atención, todo lo que usamos e ingerimos, proviene en una u otra forma del suelo o de las 
capas subyacentes, incluyendo, en la mayoría de los casos, el agua que tomamos.

Afortunadamente, este pronunciamiento de las Naciones Unidas ha marcado un hito histórico. Nunca antes se han efectuado tantas acciones de promoción sobre las funciones del suelo como en este año 2015. A lo largo y ancho del mundo y también en nuestro país, hemos expuesto, por todos los medios, estas importantes funciones.

A continuación, las enumeramos y describimos brevemente:

1. La más reconocida de todas sus funciones es, indudablemente, su rol agrícola en el sostén y el suplemento de agua y nutrimentos para la producción de cultivos que son fuente de alimentos, tejidos y biocombustibles. En la actualidad, FAO reporta que el $95 \%$ de los alimentos provienen del suelo, por lo que esta función involucra al suelo en la seguridad alimentaria de todos y cada uno de los habitantes del planeta. Esto incluye además, todos los alimentos necesarios para la producción animal. También entra en juego su importancia como fuente de fibras para la elaboración de vestimentas a pesar de la utilización de fibras sintéticas. En el caso de los biocombustibles, la relevancia del suelo está sujeta directamente a la oferta de petróleo y su importancia radica, más bien, en que su incremento constituirá una competencia de gran relevancia sobre la producción de alimentos. Pese a ello, no deja de ser interesante pensar en este tema como una alternativa en el caso de una posible crisis mundial de hidrocarburos.

2. La captura, el filtraje y el almacenamiento del agua para ser suministrada tanto a las poblaciones como a los cultivos, está principalmente en manos del suelo, de ahí que la conservación de acuíferos no es algo que pueda verse independientemente de este recurso. La mitad del ciclo hidrológico ocurre en el suelo.

3. Una de las funciones menos conocidas es que el suelo es uno de los principales reservorios dinámicos de carbono del planeta, lo que significa que dependiendo de la fracción orgánica presente en los suelos, se captura o libera carbono. Esto lo convierte en un actor de gran relevancia, y muy pocas veces considerado, en la mitigación del cambio climático. Como función ecosistémica del suelo, este aspecto es de gran impacto también en el pago de servicios ambientales. FAO estima que en términos del planeta completo, el suelo almacena 4000 billones de toneladas de carbono, en comparación con los 800 billones que acumula la atmósfera y los 360 billones de toneladas presentes en los bosques. Esto implica que en el suelo se acumula 4 quintas partes del carbono del planeta.

4. En el suelo ocurren con intensidad procesos de descomposición y reciclaje de los residuos o materiales que llegan a él, transformándolos en productos que se reincorporan al sistema natural. Una de las mayores aplicaciones de esta función es la elaboración de abonos orgánicos. No es difícil imaginar las severas limitantes a las que se enfrenta en la actualidad el suelo para poder completar esta función, ante la producción excesiva de residuos de todo tipo, provenientes de la actividad humana, que no se clasifican y en algunos casos, con excesivas cantidad de sustancias nocivas para el ambiente y la salud.

5. Otro aspecto poco conocido es el reservorio de vida y recursos genéticos que el suelo representa. La biodiversidad que existe en el suelo es tan alta como la que se puede encontrar en los bosques tropicales e igualmente representa un recurso de alto valor que debe ser preservado. Se 
dice que en una cucharadita de suelo existen más microorganismos que habitantes en la Tierra.

6. El suelo soporta todas las áreas urbanas y la infraestructura en la cual nos desenvolvemos, de ahí que dado que todas las funciones ocurren en el mismo espacio, los criterios de ordenamiento territorial y planificación del uso de la tierra deben llegar a ocupar un papel central en la agenda de los tomadores de decisiones, planificadores y políticos.

7. Finalmente, el suelo es y ha sido el encargado de preservar y resguardar toda la herencia cultural de los pueblos, según lo confirma la Arqueología, y de la historia evolutiva de la vida animal y vegetal, como lo refleja la Paleontología.

El nivel superior en donde se impulsan acciones políticas que afectan el planeta, las Naciones Unidas, desde Río 92, creó las Convenciones que han encaminado el rumbo ambiental en los últimos 20 años, a saber, la Convención de Cambio Climático de donde sale el apoyo contundente al recurso Agua y la de Biodiversidad. En ese momento, el suelo también fue considerado, sin embargo, la Convención orientada hacia este recurso se llamó "Convención de Lucha contra la Desertificación, Degradación de la Tierra y la Sequía (CNULD)". Esta convención fue poco financiada y orientada de manera sesgada hacia el problema de desertificación, que como se sabe, está prioritariamente ubicado en una cierta región del planeta.

Estas razones han ocasionado que el suelo, como recurso frágil y finito que es, haya permanecido invisibilizado ante la sociedad internacional.

El mayor esfuerzo de la comunidad científica edafológica hacia la protección y el conocimiento del recurso suelo a nivel mundial lo ha dado la Unión Internacional de la Ciencia del Suelo (IUSS por sus siglas en inglés, International Union of Soil Science). Desde hace varios años, esta sociedad científica venía trabajando la idea de construir una estrategia que permitiera enfrentar el problema de la degradación de los suelos y su conservación, e incluso había propuesto la declaración del 5 de diciembre como el Día Mundial de los Suelos. A través de reuniones preliminares y consensos, más técnicos que políticos, se lograron elaborar los documentos que dieron origen a lo que hoy se llama la Alianza Mundial por el Suelo (AMS, o GPS por su sigla en inglés, Global Soil Partnership). Sin embargo, no es sino hasta el momento en que esta iniciativa de la Alianza Mundial por el Suelo se canaliza bajo el amparo de la FAO en el 2012, que se logra concretar.

La AMS tiene la cualidad de haber logrado conjuntar lo técnico, lo político y otros actores no gubernamentales bajo una misma idea; esa parece ser la razón fundamental de su acción positiva y su posicionamiento mundial.

"La AMS es una iniciativa voluntaria y no crea derechos u obligaciones jurídicamente vinculantes entre sus miembros, ni para cualquier otra entidad, en virtud del derecho nacional o internacional. Se remite al Principio 2 de la Declaración de Rio sobre el Medio Ambiente y el Desarrollo, el cual establece que de conformidad con la Carta de las Naciones Unidas y con los principios del derecho internacional los Estados tienen el derecho soberano de explotar sus recursos en aplicación de su propias políticas ambientales y de desarrollo, así como la obligación de asegurar que las actividades que se lleven a cabo dentro de su jurisdicción o bajo su control, no perjudiquen al medio ambiente de otros Estados o de zonas situadas fuera de toda jurisdicción nacional. El mandato de la Alianza es mejorar la gobernanza de los limitados recursos de suelos del planeta a fin de asegurar suelos sanos y productivos para un mundo que goce de seguridad alimentaria, así como apoyar otros servicios ecosistémicos esenciales en consonancia con el derecho soberano de cada Estado sobre sus recursos naturales." 
La AMS surge entonces, con el propósito de convertirse en una asociación interactiva que aumentará la sensibilización, contribuirá al desarrollo de capacidades, aprovechará los mejores conocimientos y tecnologías científicas disponibles, facilitará el intercambio entre las partes interesadas y contribuirá a la gestión y utilización sostenible del recurso suelo.

En este momento existen 184 agrupaciones adscritas a esta Alianza, entre las cuales hay sociedades técnicas, universidades, centros de investigación, entre otros, y de las cuales 32 están ligadas con Latinoamérica.

Por Costa Rica destaca como miembro directo, la Asociación Costarricense de la Ciencia del Suelo (ACCS) y junto a ella todo el grupo de profesionales del Centro de Investigaciones Agronómicas (CIA) de la Universidad de Costa Rica, que han participado de la investigación y enseñanza de la edafología en el país desde hace 60 años.

La AMS con la colaboración de su Grupo Técnico Intergubernamental de Suelos (GTIS, el cual está compuesto por 27 científicos representantes de todo el mundo, uno de los cuales, en su primera designación fue de Costa Rica) ha desarrollado un documento sobre el estado del recurso suelo en el mundo que se dará a conocer al final de este mismo año, y ha logrado concretar los planes de acción para los 5 pilares que se establecieron inicialmente en la Asamblea General de la AMS. Estos 5 pilares de acción fundamentales son:

Pilar 1: Promoción del manejo sostenible del recurso suelo y mejoramiento de la gobernanza para la protección del suelo y su productividad sostenible.

Pilar 2: Fomento de la inversión, la cooperación técnica, las políticas, la concientización, la educación, la capacitación y la extensión sobre los suelos.

Pilar 3: Promoción de la investigación y el desarrollo edafológico focalizado y centrado en las brechas y prioridades que se hayan identificado y las sinergias con acciones relacionados con la producción, desarrollo ambiental y social.

Pilar 4: Mejoramiento de la cantidad y la calidad de los datos e información edafológica: recolección de datos (generación), análisis, validación, presentación de informes, monitoreo y su integración con otras disciplinas.

Pilar 5: Armonización de los métodos, medidas y los indicadores para el manejo sostenible y la protección del recurso suelo.

Con el fin de volver realmente operativos los planteamientos que se han establecido en los diferentes pilares, la actividad se ha consolidado por regiones en las que, representantes directos de los sistemas estatales de cada país, denominados puntos focales, constituyen el vínculo para hacer operativos los planes de cada pilar.

En nuestro caso, Costa Rica pertenece a la Alianza Regional de Centroamérica, México y el Caribe, y para este momento, ya está elaborado el Plan Operativo para cada uno de los pilares con fechas de ejecución que contemplan los próximos 2-3 años y en los cuales participamos representantes locales.

En Costa Rica, la celebración del Año Internacional del Suelo ha contemplado una diversidad de actividades con las cuales se ha buscado contribuir al posicionamiento del recurso suelo en cuatro escenarios fundamentales: armonizando el discurso entre los profesionales de suelos, a nivel político, en la agenda de desarrollo sostenible nacional, en la población en general y en el corazón de las futuras generaciones.

Primero que todo resultó imprescindible armonizar el discurso entre las y los científicos de la ciencia del suelo o "sueleros", que aunque no requeríamos motivación para reconocer la importancia del suelo, tenemos la responsabilidad de trasmitir un mensaje claro, congruente y convincente de lo que el recurso representa para la vida en nuestro planeta. En este sentido, durante el 2015, en primer lugar se realizó el VIII Congreso Nacional de Suelos con la participación de 250 profesionales bajo el lema "Los suelos, una 
base sólida para la vida". Se presentaron 7 charlas correspondientes a cada una de las 7 funciones del suelo a cargo de especialistas de reconocimiento mundial. El presidente de la IUSS, Dr. Rainer Horn, nos habló de agua; el Vicepresidente de esa misma Sociedad, Dr. Flavio Camargo, profundizó en las capacidades de biorremediación dentro del papel de reciclaje de los suelos; la Dra. Diana Wall, como Coordinadora de la Alianza Global de la Biodiversidad del Suelo, ahondó en el tema microbiológico; el Dr. Rattan Lal nos suministró información sobre las capacidades de secuestro de carbono de los suelos y su influencia en el cambio climático; sobre la infraestructura vial y el urbanismo nos instruyeron el Dr. Luis Guillermo Loría del LANNAME (UCR) y el Ing. Eduardo Brenes; por su parte, el arqueólogo Ricardo Vásquez trató el papel de suelo en el resguardo de nuestro patrimonio cultural, y finalmente, el M.Sc. Gonzalo Farias, Presidente de la Sociedad Brasileña de la Ciencia del Suelo, al igual que el actual Ministro de Agricultura y Ganadería (MAG), Dr. Felipe Arauz, defendieron cada uno desde su propia visión, la indiscutible relevancia del suelo para la producción agrícola.

La segunda actividad que se llevó a cabo en este sentido fue la alianza de la ACCS con el Colegio de Ingenieros Agrónomos, para realizar 4 conversatorios en las filiales de las Regiones Brunca, Huetar Norte, Huetar Caribe y Chorotega, donde se discutieron las ya mencionadas funciones ecosistémicas del suelo. También, se repasaron las características de los suelos de la región respectiva y se estableció un diálogo entre los presentes sobre las mayores limitantes edáficas en cada zona y sus posibles soluciones. Esta información sobre el estado de los suelos a nivel nacional se recopiló como insumo para futuras acciones.

A nivel político, se comenzó con un panel sobre "El ordenamiento territorial en Costa Rica" en el que se puso sobre la mesa la manera en la que las diferentes funciones del suelo compiten por el uso del mismo territorio; se discutió sobre las opciones para que éstas convivan apropiadamente y permitan la conservación del recurso.
También, se firmó la "Declaración de San José" por autoridades gubernamentales y representantes internacionales de suelos como testigos. Este documento permitió que como ACCS y como país nos comprometiéramos, cada uno desde su ámbito de acción, a defender el recurso suelo y continuar trabajando por su buen manejo y su preservación.

Finalmente, la tercera acción a nivel político, fue lograr que MINAE (Ministerio de Ambiente y Energía) y MAG asumieran el compromiso de formular una directriz ministerial conjunta, que inste a todas las instituciones $y$ usuarios del recurso suelo, al cumplimiento de los compromisos nacionales e internacionales adquiridos. Esta actividad se logró mediante el esfuerzo concertado de la ACCS y de CADETI (Comisión Asesora Sobre Degradación de Tierras que representa a la Convención de las Naciones Unidas de Lucha Contra la Desertificación en el país) en un Día de Campo y en presencia de más de 100 agricultores.

En el tema de la concientización de la población se ha hecho presencia en los diversos medios de comunicación refiriéndose a las funciones del suelo. Asimismo, los profesionales del CIA y de la ACCS incorporaron el tema en su labor cotidiana apoyándose en signos externos diseñados para la ocasión. También se colaboró ampliamente en el Cuaderno Nuestra Finca 2016 que, como todos los años es un documento que es distribuido por el MAG entre un gran número de agricultores.

Dos actividades muy particulares y en las que se buscó incidir en un grupo de la población muy diferente, fueron el billete de lotería dedicado al Año Internacional de los Suelos que elaboró la Junta de Protección Social de Costa Rica, y el Matasellos que circuló Correos de Costa Rica en un sobre diseñado par tal efecto.

También, el arte ha estado presente en esta celebración del Año Internacional de los Suelos, pues con ayuda del grupo de Recursos Naturales y en celebración del 60 aniversario del CIA se organizó un "Taller de Pintura con Suelo" que 
impartió la artista Duvi Carranza a un grupo del Programa Integral de Adulto Mayor.

Finalmente, y conscientes de la clara vinculación que debemos establecer con el sector educativo del país, se han realizado otra serie de actividades que incluyen un concurso de fotografía para muchachos, la ejecución de giras técnicas que los miembros de la ACCS ofrecieron al colegio de los muchachos ganadores para compartir con ellos las características de los diferentes suelos de la Región Este del Valle Central, y clases sobre el uso del Mapa Digital de Suelos de Costa Rica para estudiantes de un Colegio Técnico Profesional con orientación agropecuaria.

Finalmente, se llegó al nivel escolar ofreciendo charlas educativas sobre el rol del suelo en el ciclo hidrológico y en la erosión, y sobre la importancia del compostaje para mantener una buena salud de los suelos. Estas actividades se realizaron en colaboración con el Programa de Cosecha de Agua que propicia la ASADA de Paso Ancho-Boquerón, y el grupo de Recursos Naturales del CIA, apoyados por la ACCS y destacando el 60 Aniversario del CIA. Se diseñó material infográfico específico para tal efecto.

Es fundamental que todos tengamos al recurso suelo en nuestros afectos. Es parte de nuestro arraigo y ha sustentado nuestra existencia durante toda la vida. Para poder protegerlo y manejarlo bien, lo importante es que lo conozcamos, lo comprendamos y lo aprendamos a querer. $\mathrm{Y}$ eso es esencialmente lo que debemos trasmitir a nuestros niños, porque todo aquello que se quiere, se cuida y se protege permanecerá con nosotros.

Solo con niños del presente amantes y conocedores del recurso, tendremos adultos ambientalmente sensibles a los suelos del futuro. 
